\title{
Axon Regeneration Across the Site of Injury in the Optic Nerve of the Newt Triturus pyrrhogaster*
}

\author{
L.J. Stensaas and E.R. Feringa ** \\ Department of Physiology, University of Utah College of Medicine, Salt Lake City, Utah, USA
}

\begin{abstract}
Summary. The process by which axons regenerate following a freeze injury to the optic nerve of the newt was analyzed by light and electron microscopy. Freezing destroys cellular constituents in a one millimeter segment of the nerve, leaving intact the basal lamina and the blood supply to the eye. No axons are seen at the site of injury one to seven days post lesion. This contrasts with the persistence of normal-appearing but severed unmyelinated axons within the cranial stump which thus give a false appearance of early regeneration. The first axon sprouts traverse the lesion and enter the cranial stump by ten days. The number of regenerating axons increases rapidly thereafter with no signs of random growth at the site of injury. These axon sprouts tend to be somewhat larger than normal unmyelinated axons and contain dense core vesicles and abnormal organelles similar to those in growing axons in tissue culture. The persisting basal lamina inside the optic sheath appears to provide continuity across the site of injury, to orient axon sprouts, and to favor an orderly process of axon regeneration without neuroma formation.
\end{abstract}

Key words: Axon regeneration - Optic nerve - Triturus pyrrhogaster Light and electron microscopy.

\section{Introduction}

The ability of axons in the optic nerve of amphibians to regenerate stands in striking contrast to an inability of axons to regenerate in the optic nerve and

Send offprint requests to: Dr. L.J. Stensaas, Department of Physiology, 4C202 University Medical Center, University of Utah, Salt Lake City, Utah 84112, USA

* The authors wish to express their gratitude to Barbara Heindel and Jill Jones for extremely helpful technical assistance. This work was supported by grants NS 10864 and NS 05666 from the U.S. Public Health Service and by the Medical Research Service of the Veterans Administration

** Present Address: Departments of Neurology and Pathology, Veterans Administration Hospital and University of Michigan Medical Center, Ann Arbor, Michigan, USA 
other parts of the central nervous system of reptiles, birds and mammals (Gaze, 1960). Earlier studies of the regenerative process in the frog (Sperry, 1944) and the newt (Sperry, 1943) following optic nerve transection indicated anatomical and functional regeneration to appropriate portions of the midbrain after approximately one month. Reports of recent investigations indicate that the initial stages of regeneration occur considerably earlier. Axons traverse the site of optic nerve injury in only two weeks, according to ultrastructural studies of the regenerating Xenopus tadpole (Reier and Webster, 1974). This is comparable to the time required for regenerating axons in a mammalian peripheral nerve to bridge the transection site when the stumps are well apposed (Young, 1942).

Remarkably rapid growth of axons in the optic nerve of the newt following surgical transection has been reported by Turner and Singer (1974b). Nerve fibers traverse the lesion and grow into the distal stump in only four days in adult Triturus viridescens. Although the rapidity of this regeneration is exceptional for an injury involving a gap, it is in accord with studies of regeneration following interruption of axons in a peripheral nerve by crushing (Zapata et al., 1976). The time required for nerve fibers to regenerate after a nerve crush is known to be considerably less than that needed after a nerve transection. This may be due in part to alignment of the sprouts by components of the nerve whose orientation is unaffected by the crush (Sunderland, 1968). Rapid regeneration after localized freezing of a nerve has similarly been attributed to efficient fiber orientation by persisting connective tissue elements (Mira, 1972).

The present study considers the temporal aspects of the regenerative process in the optic nerve of the newt and compares events at the site of injury with those in the cranial stump. Freezing the nerve completely interrupts nerve fibers but does not destroy the optic sheath, the basal lamina of the optic nerve, or the blood supply to the retina. Under these conditions, the first regenerating axons bridge the gap in ten days. This is followed by a regular increase in axon number without signs of concomitant early degeneration.

\section{Methods}

\section{Surgical Procedure}

Adult Triturus pyrrhogaster were obtained from Valley Pet Supply, Hayward, California. At the time of the operation, they were anaesthetized by immersion in a $4 \%$ urethane solution. The optic nerve was approached through the roof of the mouth and the axons interrupted by freezing the nerve. The tips of Dupont No. 6 watchmaker's forceps which had been immersed in liquid nitrogen were inserted rapidly through the oral mucosa on either side of the nerve, and the fold of tissue containing the nerve was simultaneously frozen and compressed (Fig. 1). Thawing of the tissue required 15 to $20 \mathrm{~s}$, after which the forceps were removed and the animals allowed to recover from the anaesthetic. The animals were then divided into two groups and kept at different temperatures. Animals maintained at $25^{\circ} \mathrm{C}$ were allowed to survive 4,10 , and 14 days while those kept at $18^{\circ} \mathrm{C}$ were allowed to survive $2,7,21$ and 31 days. They were fed with brine shrimp two or three times per week.

\section{Tissue Preparation}

Animals were given an intraperitoneal injection of 100 units of heparin and anaethetized one hour later by immersion in a $4 \%$ urethane solution. They were then fixed by intracardiac perfusion for 


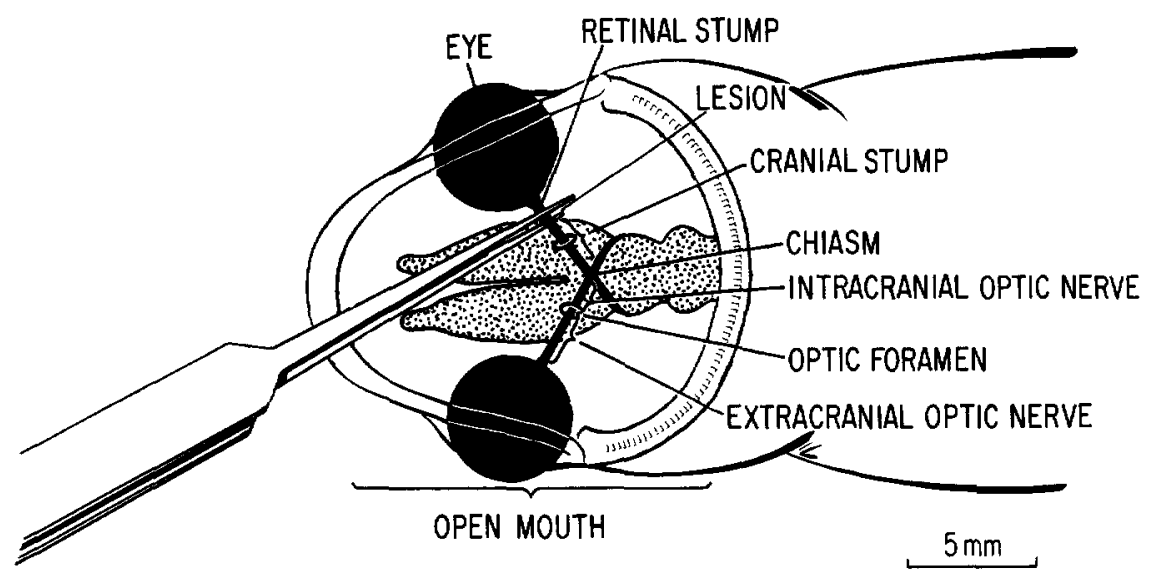

Fig. 1. Ventral view of the newt visual system (black) illustrating the location of the lesion and the various portions of the optic nerve

7-10 min at a pressure of $260 \mathrm{torr} ; 300-500 \mathrm{ml}$ of fixative were used per animal. The perfusate consisted of $0.1 \mathrm{M}$ phosphate buffer, $5 \%$ glutaraldehyde, $1 \%$ paraformaldehyde and 0.01 calcium chloride. Following perfusion, the brain was exposed, the anterior portion of the eyes removed and the body placed in cold fixative for $12-18 \mathrm{~h}$. The eyes and attached optic nerve were then removed and placed in buffered $2 \%$ osmium tetroxide for four hours. Intracranial portions of the optic nerves and chiasm were also removed and osmicated for four hours. All tissue was dehydrated in ethanol, embedded in araldite, and the plastic partially polymerized to yield blocks whose consistency facilitated "free-hand" sectioning with a razor blade. Intracranial portions of the right and left optic nerves near the chiasm, approximately $1 \mathrm{~mm}$ cranial to the site of injury, were trimmed to provide a face exactly normal to the axis of the nerve. The blocks were then hardened, and semithin sections were cut, mounted on glass slides and stained with methylene blue. Ultrathin sections were cut either at the same levels, or longitudinal to the axis of the nerve. They were mounted on formvar film in one hole $(1 \times 2 \mathrm{~mm})$ grids, and stained with uranyl acetate and lead citrate.

Additional free-hand sections of the extracranial portion of the optic nerve were used to identify the site of the lesion and to orient the tissue for transverse sections. Semithin and ultrathin sections of the site of injury and from adjacent portions of the retinal stump were then prepared.

\section{Analytical Procedures}

The incidence of nerve fibers for each optic nerve was estimated by systematically scanning a section of the nerve with the electron microscope at a magnification of $7800 \times$, locating that portion of the nerve with the highest concentration of nerve fibers, and counting all the fibers within the field provided by a $10 \mathrm{~cm}$ circle on the screen. Unmyelinated nerve fibers were designated rare when there were only 2-3 per field, common when up to 15 or 20 were found per field, and abundant when $30-50$ could be counted in the standard area. Myelinated axons were classified as rare when there were 1-2 per field; common when there were 3-4; abundant when there were 5-7, and normal when there were 8 or more in the given area.

The size of unmyelinated nerve fibers was determined by the following procedure. An optic nerve was selected at each time interval and a montage was prepared at a total magnification of $20,000 \times$ which extended from the pial surface through the center of the nerve to the opposite pial surface. Where axons were few, the entire montage was searched for axons and all were measured. A central strip was selected in montages containing numerous axons, and all axonal profiles lying wholly or partially within its limits were measured. The width of this strip varied from 1.5 to $6 \mathrm{~cm}$ to include 500 or more axons. 
To avoid overestimating the size of individual nerve fibers whose irregular trajectory through the nerve resulted in elongate rather than round profiles, the minimum diameter, measured to the nearest $0.1 \mu \mathrm{m}$, was used as a measure of axon size. Histograms were constructed, showing the percent of counted fibers which fell into size ranges from $<0.1$ to $>0.8 \mu \mathrm{m}$. All myelinated axons in this same area of the montage were counted; but, because of their scarcity and widely varying diameter, they were lumped in the histograms in the $>0.8 \mu \mathrm{m}$ group.

\section{Results}

The optic nerve of adult $T$. pyrrhogaster normally displays myelinated and unmyelinated axons in a ratio of approximately 1:40. Although the size of the optic nerve varies, it usually contains $800-1000$ myelinated axons and $30-40,000$ unmyelinated axons. A few degenerating myelinated axons are generally present in a given cross section of the normal optic nerve, but signs of unmyelinated axon degeneration were not observed. Freezing a $1 \mathrm{~mm}$ segment of the extracranial portion of the nerve produces a short $(0.5-1.0 \mathrm{~mm})$ retinal stump and a somewhat longer (1.0-1.5 mm) cranial stump (Fig. 1).

\section{Light Microscopy}

Total necrosis occurs in the optic nerve at the site of injury. At seven days, this portion of the nerve contains macrophages as its only viable cellular constituent (Fig. 2). The optic sheath remains intact, but there is shrinkage of necrotic nerve tissue. Within the cranial portions of the cranial stump glial constituents appear normal, and the contour of the nerve is unchanged, but signs of myelin destruction are apparent (Fig. 5).

At 14 days, the diameter of the injured nerve is appreciably smaller. The only cells in the frozen portion of the nerve are numerous macrophages with dark somata (Fig. 3). The periphery of the nerve is occupied by a rim of regenerating nerve fibers. The cranial stump of the nerve is reduced in size and frequently contains more cell nuclei per section than normal (Fig. 6). Degenerating myelinated axons occur throughout the nerve.

At 21 days, the injured nerve contains few cell somata, numerous unmyelinated axons, and no myelinated axons (Fig. 4). Glial cells near the retinal and cranial margins of the injured segment display signs of hypertrophy. The increasing diameter of the cranial stump at 21 days post lesion is due to the large number of regenerating axons (Fig. 7). They form a zone near the surface of the nerve in which glial cells and remnants of degenerating myelinated axons are relatively rare. A few small myelinated axons can be observed near the center of some nerves.

\section{Electron Microscopy}

Axons do not persist in the injured segment seven days post lesion (Fig. 8). Macrophages are the only viable cells within the necrotic zone, and most contain 

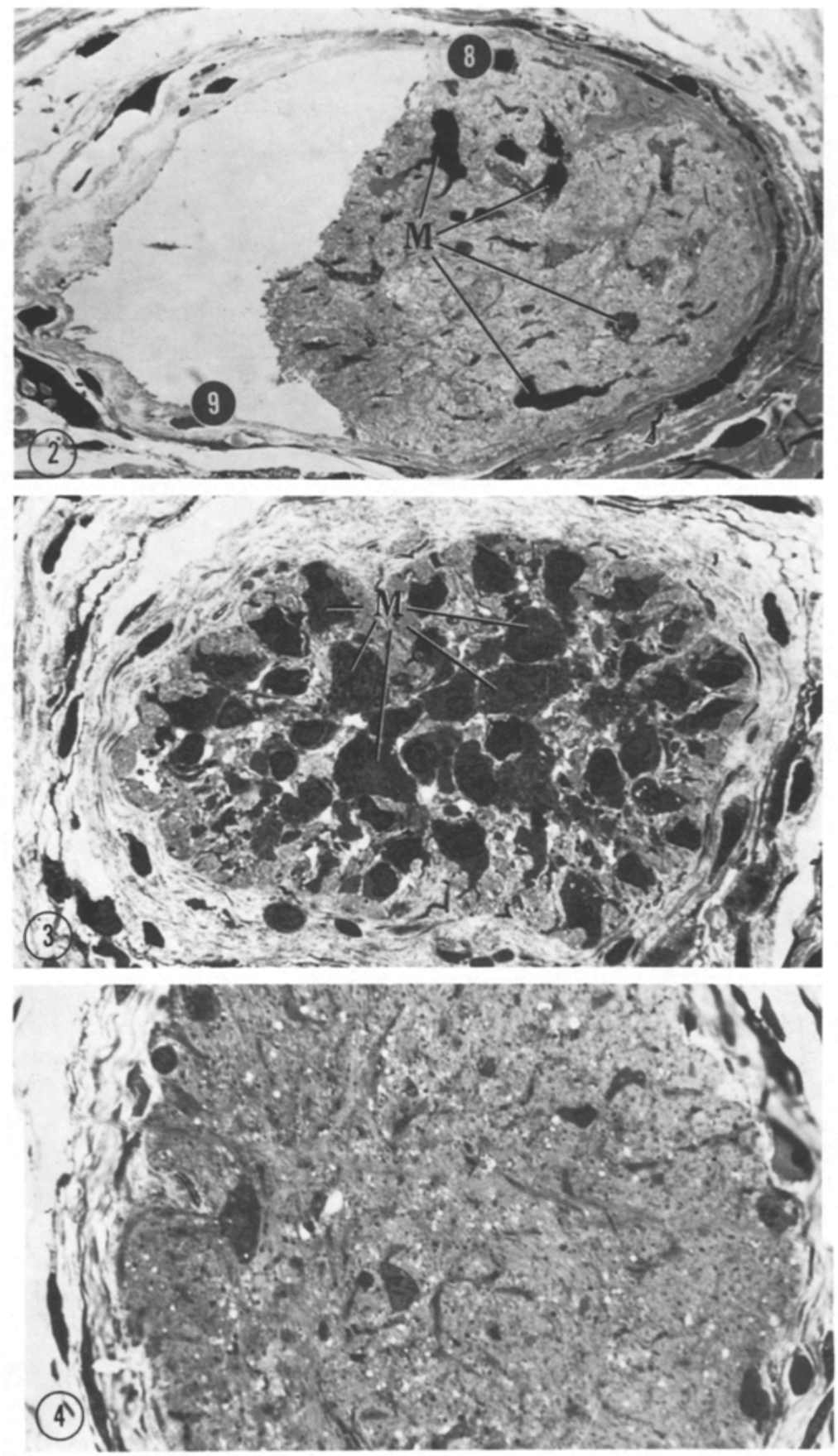

Figs. 2-4. The extracranial site of optic nerve injury 7, 14, and 21 days post lesion illustrating stages of necrosis (Fig. 2), phagocytosis (Fig. 3), and axon regeneration (Fig. 4). The approximate location of electron micrographs in succeeding figures is indicated by circles with numbers. Macrophage $(M)$. $\times 425$ 


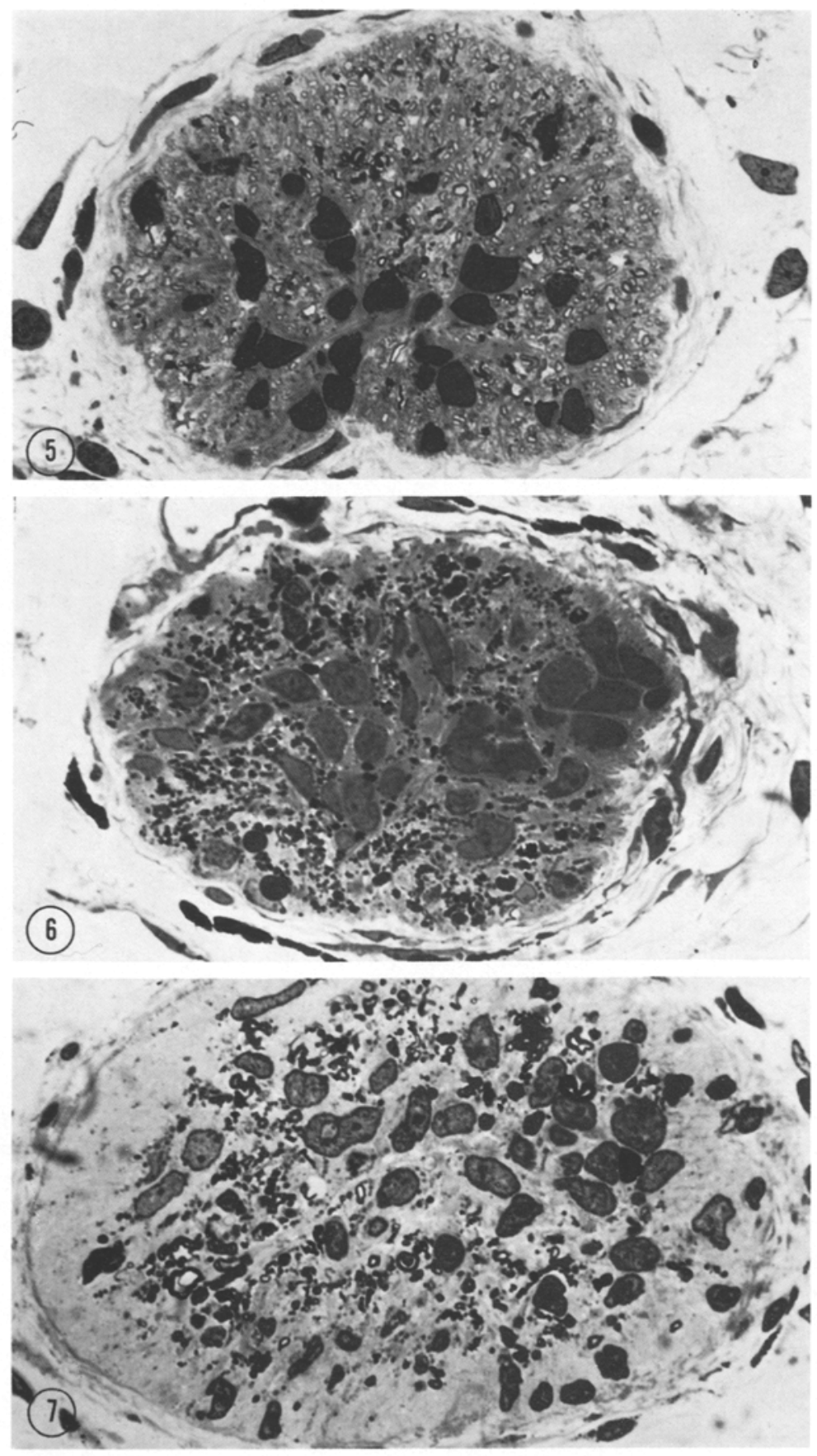

Figs. 5-7. Cranial stump of the optic nerve 7, 14, and 21 days post lesion. Nerve shrinkage and loss of myelinated axons at 7 (Fig. 5) and 14 days (Fig. 6) is followed by an increase in diameter at 21 days (Fig. 7 ) due to unmyelinated axon regeneration. $\times 425$ 

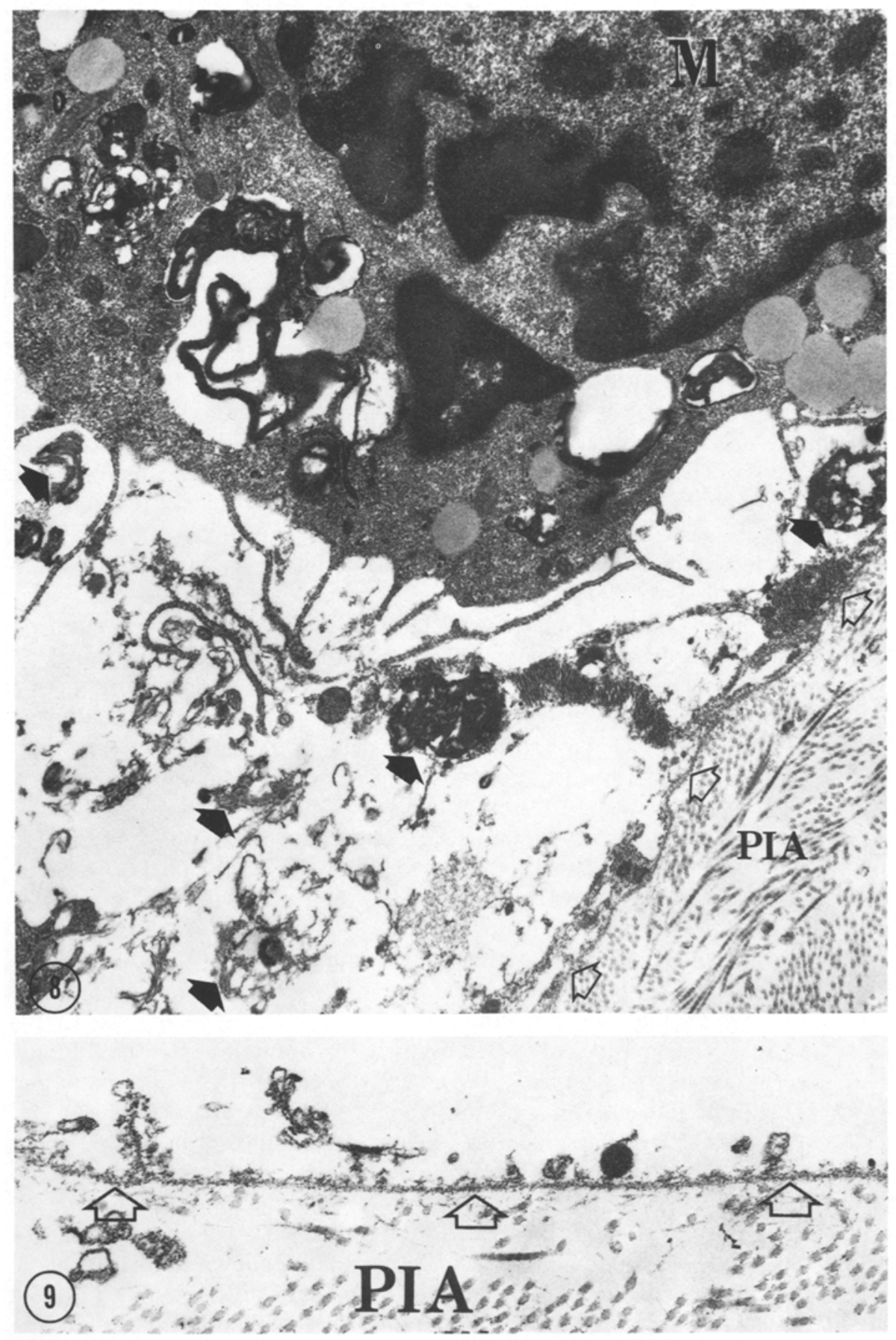

Fig. 8. The extracranial site of injury 7 days post lesion contains cellular debris (black arrow), macrophages $(M)$, and an intact basal lamina (open arrows) but no axons. Note myelinated axon debris inside the macrophage. $\times 14,850$

Fig. 9. The basal lamina (open arrows) at the pial surface (PIA) of the nerve above remains intact despite shrinkage and withdrawal of axonal debris. $\times 34,500$ 
remnants of degenerating myelinated axons. Axonal debris is confined within the basal lamina. The latter remains intact as a continuous lining for the optic sheath even following withdrawal of necrotic material from areas formerly occupied by the nerve (Fig. 9). Seven days post lesion the cranial portions of the nerve contain a mixture of degenerating unmyelinated and myelinated axons together with apparently normal nerve fibers (Fig. 10). Their incidence in the cranial stump is summarized in Table 1. No axonal profiles containing dark core vesicles or other unusual organelles indicative of growth were seen at this time.

Bundles of axons are first visible at the site of injury ten days post lesion (Fig. 11). The regenerating axon sprouts are concentrated near the pial surface and confined within the basal lamina. No nerve fibers are observed in connective tissue of the optic sheath, and few axons occur in association with macrophages near the center of the nerve. Remnants of myelinated axons are recognizable within macrophages, but there is little or no extracellular debris at this stage. Astrocyte processes extend from the retinal stump into the site of injury. They are less numerous than axons except near the boundary between injured and non-injured portions of the nerve, where they can be seen to arise from large reactive astrocytes. Within the lesion, processes of astrocytes are longitudinally oriented and are frequently separated from axon sprouts by macrophage processes. Oligodendrocytes were not observed at the site of the injury, but they do persist as normal-appearing, unreactive elements in both retinal and cranial stumps. The cranial stump at ten days consists primarily of astrocyte processes, but extensions of oligodendrocytes and microglial cells are also visible (Fig. 12) Reactive astrocytes grow out to form a feltwork of complex, interdigitated cells and processes with little intracellular space. Axons are few in number (Table 1) and scattered throughout the nerve. They frequently contain dense core vesicles and clear vesicles (Fig. 12). A few degenerating unmyelinated axons are visible in the retinal stump of those nerves showing the greatest number of axons (Table 1).

Twenty-one and 31 days post lesion, unmyelinated nerve fibers occur throughout the nerve (Figs. 13, 14). The observed variability in nerve fiber regeneration at these periods (Table 1) is probably related to differences in the size of the lesion and to normal biological variability between animals. A few myelinated axons were noted in some nerves. Axons are confined by the basal lamina, and accessory axon bundles in the optic sheath have not been observed. Clear and dense core vesicles, lysosomes, membranous reticulum, and other organelles are common, especially in large axon profiles. Processes of reactive astrocytes extend into the site of injury but are greatly outnumbered by the neural elements. Astrocytes in the cranial stump near the chiasm display few reactive changes.

Measurements of individual unmyelinated axons are shown as histograms in Figure 15: (1) from the normal optic nerve contralateral to the lesion, (2) from the injured nerve segment and (3) from the intracranial stump near the chiasm. The distribution of fiber diameters is indicated to the nearest one tenth micron. A single peak of unmyelinated axons corresponding to fibers having a diameter of $0.2 \mu \mathrm{m}$ is always seen in the normal optic nerve. The percentages of axons in the range of 0.3 to $0.8 \mu \mathrm{m}$ are variable but form part of an asymmetrical distribution pattern. The reason for the variability is not known but appears to be unrelated 


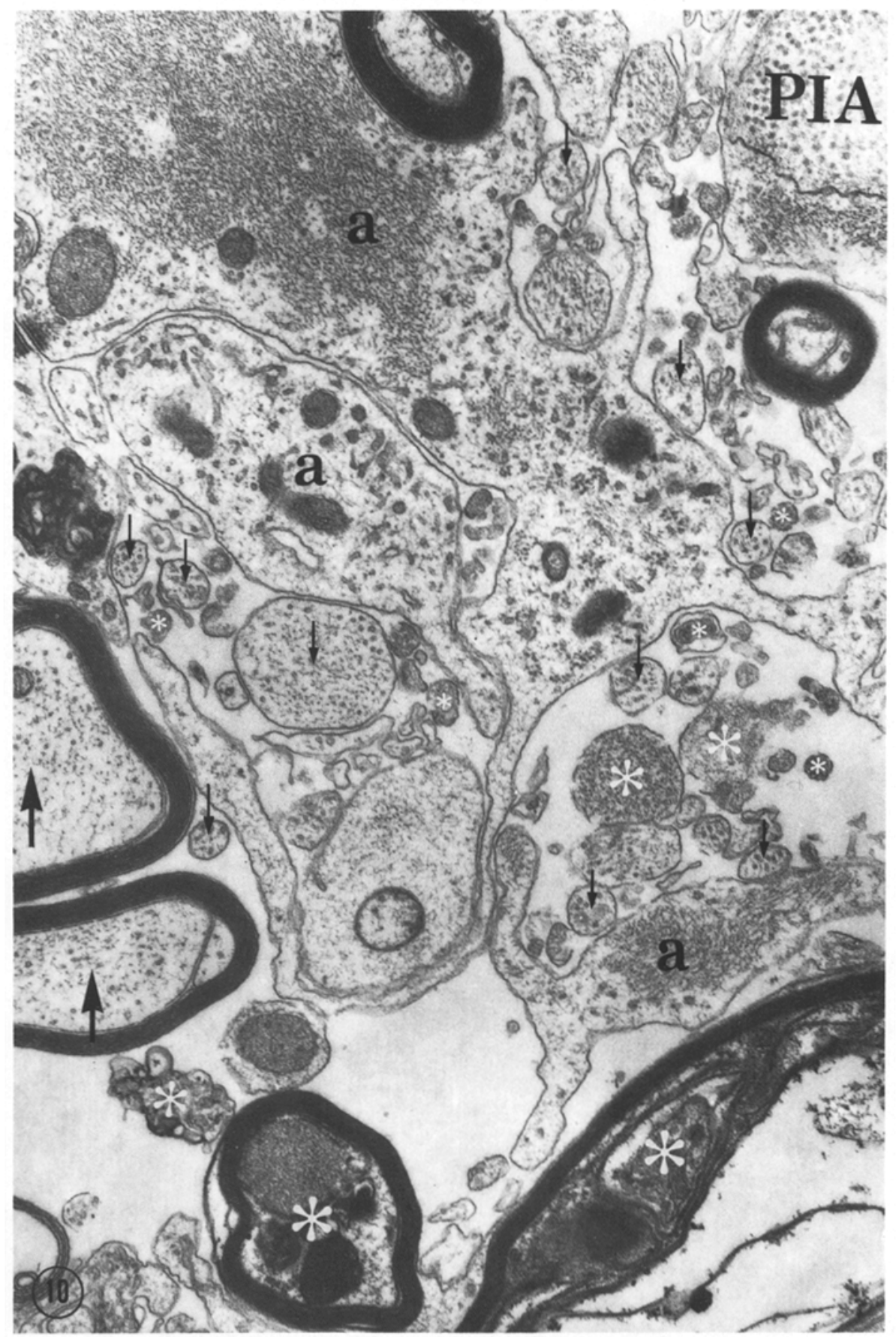

Fig. 10. Degenerating axons (white asterisk) and severed but apparently normal myelinated (large arrows), unmyelinated axons (small arrows) in the cranial stump 7 days post lesion. Astrocytes $(a)$. $\times 25,000$ 
Table 1. Incidence of myelinated and unmyelinated axons in the cranial stump of the newt optic nerve

\begin{tabular}{|c|c|c|c|c|}
\hline \multirow[t]{2}{*}{ Days post lesion } & \multirow[t]{2}{*}{ Animal number } & \multicolumn{3}{|l|}{ Cranial stump } \\
\hline & & $\begin{array}{l}\text { Degenerating } \\
\text { unmyelinated } \\
\text { axons }\end{array}$ & $\begin{array}{l}\text { Myelinated } \\
\text { axons }\end{array}$ & $\begin{array}{l}\text { Unmyelinated } \\
\text { axons }\end{array}$ \\
\hline Normal & AR 12 & 0 & ++++ & ++++ \\
\hline Normal & AR 148 & 0 & ++++ & ++++ \\
\hline 7 & $\begin{array}{l}\text { AR } 43 \\
\text { AR } 46 \\
\text { AR } 49\end{array}$ & $\begin{array}{l}+++ \\
+++ \\
+++\end{array}$ & $\begin{array}{l}++ \\
++ \\
++\end{array}$ & $\begin{array}{l}++ \\
++ \\
++\end{array}$ \\
\hline 10 & $\begin{array}{l}\text { AR } 119 \\
\text { AR } 124 \\
\text { AR } 130 \\
\text { AR } 133\end{array}$ & $\begin{array}{l}0 \\
+ \\
0 \\
+\end{array}$ & $\begin{array}{l}0 \\
0 \\
0 \\
0\end{array}$ & $\begin{array}{l}+ \\
+ \\
+ \\
+\end{array}$ \\
\hline 14 & $\begin{array}{l}\text { AR } 148 \\
\text { AR } 160\end{array}$ & $\begin{array}{l}0 \\
0\end{array}$ & $\begin{array}{l}0 \\
0\end{array}$ & $\begin{array}{l}++ \\
++\end{array}$ \\
\hline 21 & $\begin{array}{l}\text { AR } 67 \\
\text { AR } 55 \\
\text { AR } 58 \\
\text { AR } 61 \\
\text { AR } 64\end{array}$ & $\begin{array}{l}0 \\
0 \\
0 \\
0 \\
0\end{array}$ & $\begin{array}{l}0 \\
0 \\
+ \\
+ \\
0\end{array}$ & $\begin{array}{l}++ \\
+ \\
+++ \\
+++ \\
+++\end{array}$ \\
\hline 31 & $\begin{array}{l}\text { AR } 73 \\
\text { AR } 82 \\
\text { AR } 94 \\
\text { AR } 97 \\
\text { AR } 79 \\
\text { AR } 91 \\
\text { AR } 76 \\
\text { AR } 88\end{array}$ & $\begin{array}{l}0 \\
0 \\
0 \\
0 \\
0 \\
0 \\
0 \\
0\end{array}$ & $\begin{array}{l}0 \\
0 \\
0 \\
0 \\
0 \\
0 \\
+ \\
+\end{array}$ & $\begin{array}{l}+ \\
+ \\
+ \\
+ \\
++ \\
+++ \\
+++ \\
+++\end{array}$ \\
\hline
\end{tabular}

to differences in the diameter of the optic nerve. A single peak in the range of 0.2 to $0.4 \mu \mathrm{m}$ occurs in regenerating nerves as part of an asymmetrical distribution and is similar to that in the normal nerve. Fibers in the range of 0.3 to $0.4 \mu \mathrm{m}$ are somewhat more common than normal, especially at the earliest stages of regeneration. The peak fiber size tends to be centered at $0.3 \mu \mathrm{m}$ instead of $0.2 \mu \mathrm{m}$ both at the site of injury and in the cranial stump. The absence of fibers extracranially at the site of injury at seven days stands in contrast to the presence of fibers in the cranial stump. The fact that severed but normal-appearing unmyelinated axons in the intracranial portion of the nerve at seven days display a relatively normal distribution pattern, after the disappearance of well over half the nerve fibers, indicates no selectivity in the process of early axon breakdown.

Myelinated axons are rare by comparison with unmyelinated axons. All have diameters greater than $0.8 \mu \mathrm{m}$. Their number is so small in the area of the nerve from which measurements were taken that useful information could not be gained by plotting their diameter in the usual $0.1 \mu \mathrm{m}$ size increments. The large peak at seven days in the cranial stump is due to the loss of many unmyelinated axons and clustering through shrinkage of the nerve. 

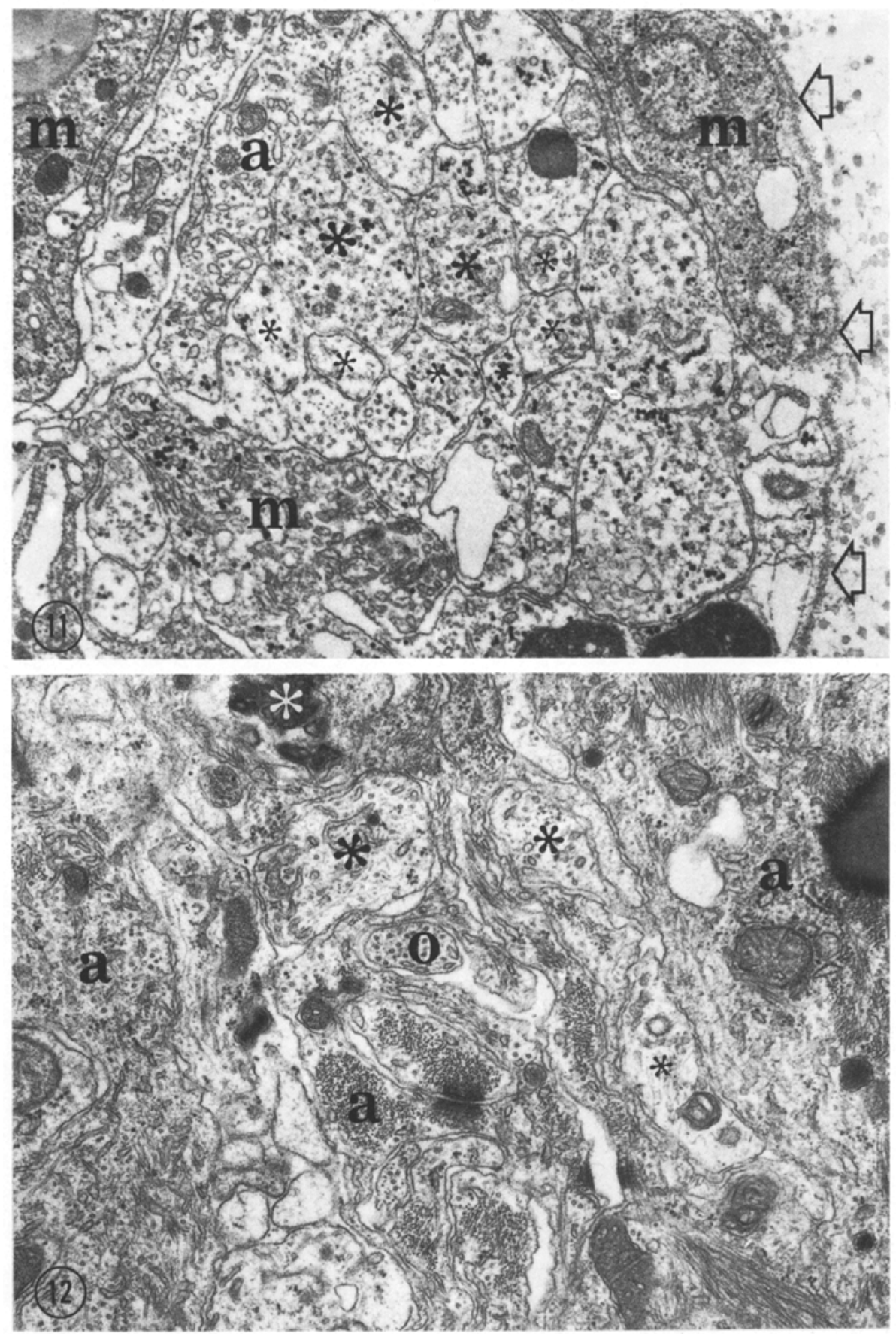

Fig. 11. Regenerating optic nerve fibers (asterisks) at the extracranial site of injury 10 days post lesion. The axons are situated beneath the basal lamina (clear arrows) of the pial surface. Macrophage $(M) . \times 25,000$

Fig. 12. The cranial stump of the optic nerve 10 days post lesion contains regenerating axons (black asterisks), oligodendrocyte $(O)$, and astrocyte processes $(a)$. Unmyelinated axon debris (white asterisks) is visible within astrocytes but does not occur in the intercellular clefts. $\times 25,000$ 

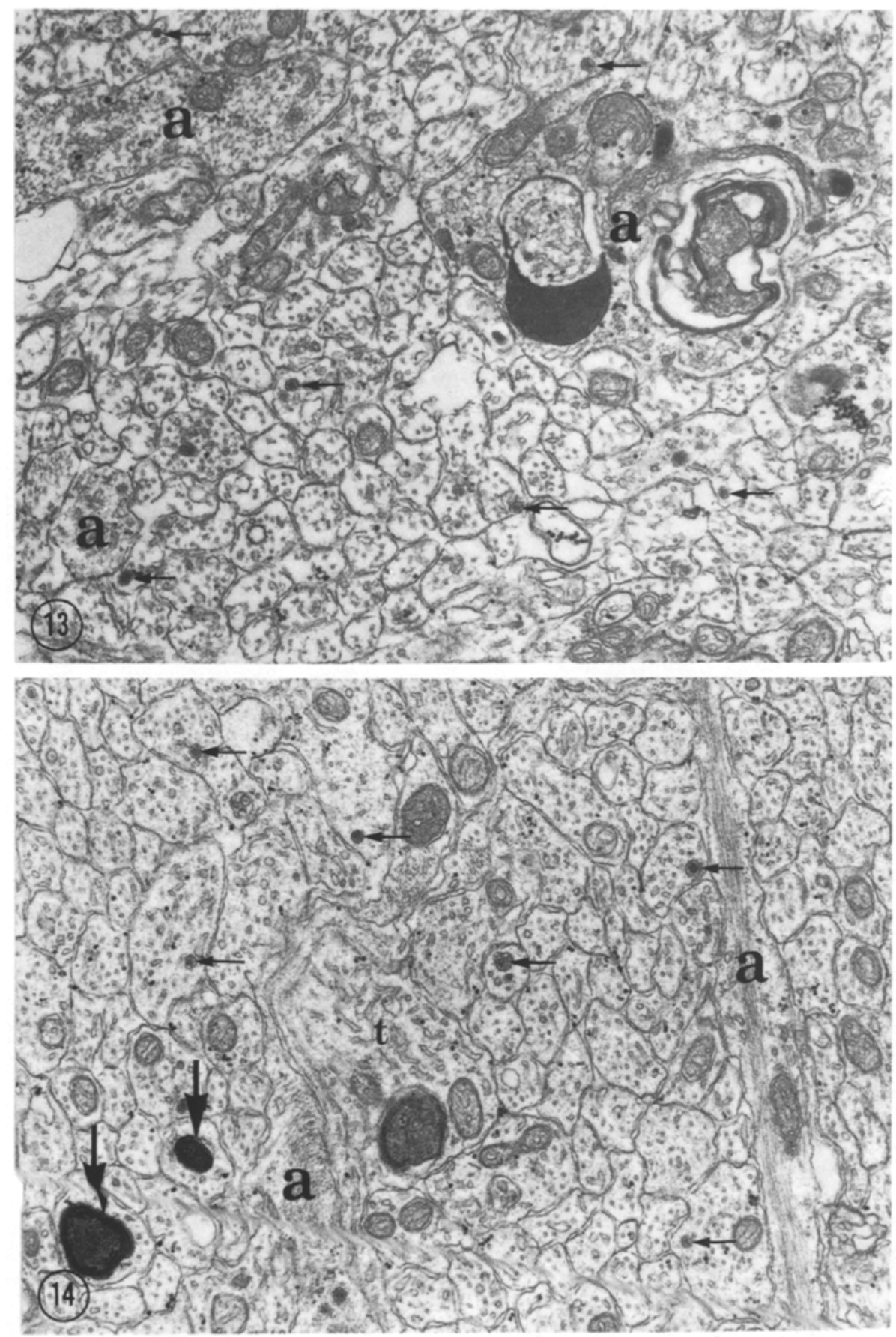

Fig. 13. The extracranial site of injury 31 days post lesion contains numerous regenerating axons and astrocyte processes (a) containing glycogen and filaments. Dense core vesicles (small arrows) characteristic of regenerating axons are visible in some of the axon profiles. $\times 25,000$

Fig. 14. The cranial stump 31 days post lesion contains regenerating nerve fibers separated by astrocyte processes $(a)$. The larger axons display dense core vesicles (small arrows), dense bodies (large arrows) and tubular reticulum (circles). $\times 25,000$ 

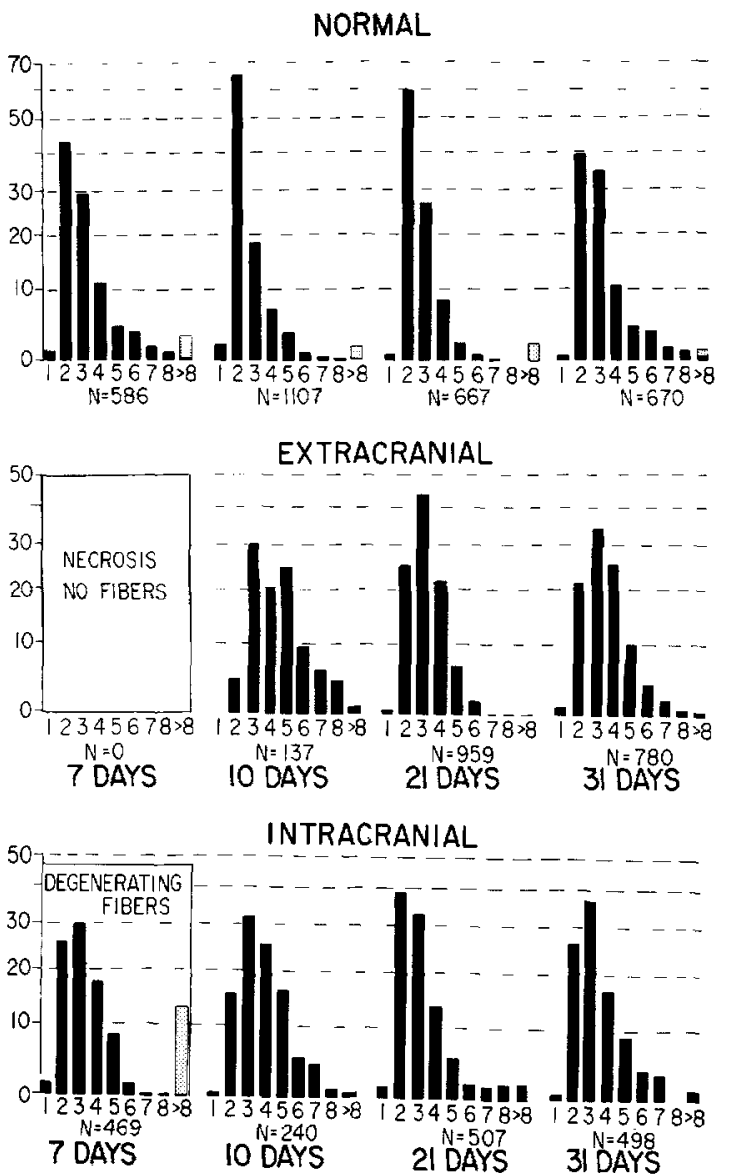

Fig. 15. Histograms of the size of unmyelinated (black) and myelinated (stippled) axons in the normal, degenerating and regenerating newt optic nerve

\section{Discussion}

\section{Guides for Regenerating Axons}

Freezing a portion of the optic nerve kills glial cells and all axons within the optic sheath. Thus, it not only leads to complete interruption of nerve fibers but also destroys the cellular constituents which provide ensheathment and support for myelinated and unmyelinated axons. The basal lamina is the only ectodermal structure surviving this form of injury, and it is this glial remnant which lines the optic sheath and provides continuity across the lesion. Regenerating axons first appear along this surface, and it is here that nerve fibers tend to congregate at early stages of growth. The apparent preference for the basal lamina may be related to its location at the surface of the nerve near the source of oxygen and other metabolites in the cerebrospinal fluid, since the normal and regenerate nerve is avascular. Alternately, the longitudinal orientation of the basal lamina 
might serve as a tube-like guide down which the sprouts can travel in order to avoid cellular debris and randomly oriented macrophages in the necrotic core of the nerve. Thus, it may constitute the only substrate for the growth of regenerating axons. Connective tissue components of the optic sheath might be supposed to serve equally well in guiding sprouts to the central stump in view of the recent study of Reier and Webster (1974). This does not occur; all regenerating nerve fibers lie within the basal lamina, do not invade the optic sheath, and show no tendency to form aberrant fiber bundles outside the nerve.

Axon growth in vivo is generally assumed to require association with appropriate glial cells (Haftek and Thomas, 1968; Egar and Singer, 1972; Egar et al., 1972). The present investigation appears to contradict this view by revealing axon sprouts lacking direct association with glial cells at the site of injury. Processes of astrocytes which grow out from the retinal stump during early stages of regeneration tend to be separated from the bundles of axon sprouts, are few in number, and generally located near the center of the nerve rather than at the periphery with the principal concentration of nerve fibers. Thus, they appear to be ill-suited to serve as templates or guides for the regenerating axons or to provide physical support and ensheathment for them.

Conversely, the failure of axon growth and functional regeneration in the mammalian central nervous system has been attributed to blocking of axon sprouts by the reactive glial cells forming a scar (Clemente, 1964; Windle, 1955). In the newt, sprouts must grow into the cranial stump which consists of a feltwork of complexly interwoven and highly differentiated astrocytes. The growing nerve fibers thus encounter conditions similar to those in a mammalian glial scar as they enter viable tissue to the distal nerve stump. Although this dense and irregular matrix of glial cells appears better adapted to impede than to assist growth of the axon sprouts, the regenerating axons readily grow within it in a well oriented fashion. Glial "channels" (Egar and Singer, 1972; Turner and Singer, 1974b), gutters (Reier and Webster, 1974), or other longitudinally oriented structures which might serve as guides could not be identified in the cranial nerve stump where the first axon sprouts seem to follow remnants of degenerating myelinated axons towards the tectum. Axons from the injured segment abandon their location at the pial surface to become distributed throughout the cranial nerve stump. The fact that they are well ordered and show few signs of random growth appears to favor the concept that good conditions for regeneration can exist among the densely-packed and highly differentiated glial cells despite their striking resemblance to the scar surrounding lesions of the mammalian central nervous system.

\section{Speed of Regeneration}

The appearance of new axons in the cranial stump ten days after injury to the optic nerve of $T$. pyrrhogaster is consistent with findings reported for the fish (Attardi and Sperry, 1963; Murray, 1976) and anurian amphibian tadpole (Reier and Webster, 1974). According to these studies, at least one to two weeks is required for axon sprouts to bridge a gap or discontinuity. It is also consistent with accounts of regeneration in peripheral nerves where a gap separates the 
two portions of the nerve. The classical studies of Cajal (1968) indicating that axon sprouts can be expected to grow at an average rate of $2.5 \mathrm{~mm}$ per day, following an initial delay (2-5 days) and a slow rate of growth through the scar ( $0.25 \mathrm{~mm}$ per day), have been confirmed by subsequent investigations (Sunderland, 1968). The close correspondence between the rate of regeneration based on these estimates and the actual period required for fibers to appear in the distal stump in the studies cited above indicates that similar principles probably apply to the early stages of regeneration in the fish and amphibian visual system.

The present study does not agree with the recent reports of Turner and Singer (1974b) of axon regeneration into the cranial stump in only four days in $T$. viridescens. The discrepancy of approximately one week required for axons to reappear in the cranial stump of a newt whose nerve fibers and glial cells are virtually identical to the species considered in the present study cannot presently be accounted for. The difference is obviously not due to an incomplete lesion since both forms of injury lead to physical interruption of all axons. On the other hand, longer rather than shorter regeneration times would be expected in the transected nerve of $T$. viridescens since a gap or physical discontinuity is known to constitute a greater impediment than lesions which leave continuity of the nerve intact (Ramon y Cajal, 1968; Young, 1942).

The discrepancy would appear to be related to the identification of severed but undegenerated unmyelinated axons as regenerating axon sprouts. Normalappearing axons persist in the cranial stump when there are no axons at the site of injury. Severed unmyelinated nerve fibers which appear normal several days after injury have been observed in other poikilothermic animals (Kruger and Maxwell, 1969), and could be mistaken for regenerating nerve fibers. Dense core vesicles and other organelles characteristic of growing axons serve to provide the important clue and the only reliable criteria to distinguish the regenerating sprouts from persisting severed axons. Such features were not observed in severed axons of the cranial stump.

\section{Fiber Size}

Unmyelinated nerve fibers predominate in the normal newt optic nerve (Gage, 1970; Turner and Singer, 1974a) and are the only axon type in the early stages of regeneration. In both normal and regenerating nerves, they represent a spectrum in which the diameter of the largest unmyelinated axons is continuous with that of a small contingent of myelinated nerve fibers. The present study provides no evidence for two distinct classes of regenerating fibers. The discontinuity in fiber spectrum between 0.5 and $0.8 \mu \mathrm{m}$ reported by Turner and Singer (1974b) was apparent neither in the cranial stump nor extracranially at the site of injury. It is of interest that the first fibers to regenerate are larger than normal. This appears to be qualitatively different from nerve fibers in the goldfish where the first fibers are small $(<0.1 \mu \mathrm{m})$ and progressively increase in diameter.

The numerous discrepancies in the ultrastructural studies of regeneration cannot be accounted for on the basis of the information presently available. The use of different species could be responsible for some differences since the pro- 
portions of myelinated to unmyelinated fibers varies greatly. Proximity of sampled axons to the lesion is a second factor which might account for different findings while the environmental temperature is a third variable which could affect axon regeneration. A solution to the problem of whether a common pattern is characteristic of the regenerative process in lower vertebrates and the extent to which glial cells influence this process obviously requires further study of material prepared from various poikilothermic animals under identical experimental conditions. These poikilothermic animals constitute, nevertheless, invaluable models for analysis of the regenerative process and may ultimately provide information regarding the nature of the impediment to axon growth in mammals.

\section{References}

Attardi, D.G., Sperry, R.W.: Preferential selection of central pathways by regenerating optic-fibers. Exp. Neurol. 7, 46-64 (1963)

Clemente, C.: Regeneration in the vertebrate central nervous system. Int. Rev. Neurobiol. 6, 257-301 (1964)

Egar, M., Simpson, S.B., Singer, M.: The growth and differentiation of the regenerating spinal cord of the lizard Anolis carolinensis. J. Morph. 131, 131-151 (1970)

Egar, M., Singer, M.: The role of ependyma in spinal cord regeneration in the urodele, Triturus. Exp. Neurol. 37, 422-420 (1972)

Gaze, R.M.: Regeneration of the optic nerve of amphibia. Int. Rev. Neurobiol. 2, 1-40 (1960)

Gaze, R.M.: The formation of nerve connections. New York: Academic Press 1970

Haftek, J., Thomas, P.K.: Electron microscope observations on the effects of localized crush injuries on the connective tissues of peripheral nerve. J. Anat. (Lond.) 103, 233-243 (1968)

Kruger, L., Maxwell, D.S.: Wallerian degeneration in the optic nerve of a reptile: an electron microscopic study. Amer. J. Anat. 125, 247-270 (1969)

Mira, J.C.: Changes in number and diameter of regenerating axons after a freeze injury to the nerve. C.R. Acad. Sci. (Paris) 275, 979-982 (1972)

Murray, M.: Regeneration of retinal axons into the goldfish optic tectum. J. comp. Neurol., in press (1976)

Ramón y Cajal, S.R.: Degeneration and regeneration in the nervous system, Vol. 1. New York: Hafner 1968

Reier, P.J., Webster, H. de F.: Regeneration and remyelination of Xenopus tadpole optic nerve fibers following transection or crush. J. Neurocytol. 3, 591-618 (1974a)

Reier, P.J., Webster, H. de F.: Remyelination in the regenerating optic nerves of Xenopus tadpoles. Anat. Rec. 178, 446 (1974b)

Sperry, R.W.: Visuomotor coordination in the newt (Triturus viridescens) after regeneration of the optic nerve. J. comp. Neurol. 79, 33-55 (1943)

Sperry, R.W.: Optic nerve regeneration with return of vision in anurans. J. Neurophysiol. 7, 57-69 (1944)

Sunderland, S.: Nerves and nerve injuries. Baltimore: Williams and Wilkins 1968

Turner, J.E., Singer, M.: An ultrastructural study of the newt (Triturus viridescens) optic nerve. J. comp. Neurol. 156, 1-18 (1974a)

Turner, J.E., Singer, M.: The ultrastructure of regeneration in the severed newt optic nerve. J. exp. Zool. 190, 249-268 (1974 b)

Windle, W.F.: In: Regeneration in the central nervous system (W.F. Windle, ed.). Springfield, Illinois: Thomas 1955

Young, J.Z.: Functional repair of nervous tissue. Physiol. Rev. 22, 318-374 (1942)

Zapata, P., Stansaas, L.J., Eyzaguirre, C.: Axon regeneration following a lesion of the carotid nerve: electrophysiological and ultrastructural observations. Brain Res., in press (1976) 